\title{
Weighted Multiple Model Adaptive Control of Time-varying Systems*
}

\author{
Weicun Zhang ${ }^{1} \dagger$ Qing $\mathbf{L i}^{2}$ \\ ${ }^{1}$ School of Automation and Electrical Engineering \\ University of Science and Technology Beijing \\ Beijing 100083, China \\ E-mail: weicunzhang@ustb.edu.cn \\ ${ }^{2}$ School of Automation and Electrical Engineering \\ University of Science and Technology Beijing \\ Beijing 100083, China \\ E-mail:qingli@ies.ustb.edu.cn
}

\begin{abstract}
This paper is concerned with the weighted multiple model adaptive control (WMMAC) of stochastic plant with time-varying parameters, such as slowly time-varying plant or parameter jump plant. An improved weighting algorithm is proposed for this kind of control systems. The effectiveness (sharp and correct 0-1 convergence) of the weighting algorithm and the robust stability of the resulting closed-loop WMMAC system has been verified through many simulation results.
\end{abstract}

Keywords: Stability, Convergence, Weighting algorithm, Multiple model adaptive control.

\section{INTRODUCTION}

As we know, weighting algorithm plays an important role in WMMAC systems. According to reference [1], the closed-loop stability of a WMMAC system mainly depends on three conditions: first, the model set includes the true model of the plant or a closet one to the plant; second, the weighting algorithm converges correctly; third, each local controller stabilizes its corresponding model. Besides it is obvious that the convergence rate of a weighting algorithm is related to the transient process of the WMMAC system.
In classical weighted multiple model adaptive estimation (WMMAE) and WMMAC ${ }^{[2-8]}$, as well as robust multiple model adaptive control (RMMAC) ${ }^{[9-11]}$, probabilistic weighting algorithm was adopted, which is based on multiple Kalman filters, dynamic hypothesis testing, and Bayes' theorem. Some convergence results on the probabilistic weighting algorithm have been obtained ${ }^{[12-15]}$. In [10], Fekri, Athans and Pascoal pointed out that under certain ergodicity and stationarity assumptions, one of the posterior probabilities will converge with probability 1 to unity and will 'identify' the model closest to the true plant, i.e. the one with smallest

\footnotetext{
${ }^{*}$ This work was supported by Major State Basic Research Development Program (973 Program) (No. 2012CB821200) and National High-Tech Research and Development Program of China (863 Program) (No. 2011AA060408).

$\dagger$ Corresponding author.
}

Published by Atlantis Press

Copyright: the authors 
Baram Proximity Measure (BPM) $)^{[10,12]}$.

However, since using Kalman filters to drive the hypothesis testing, the classical WMMAC scheme can suffer from poor performance due to either large initial state estimate error or inaccurate knowledge of the disturbance/noise statistics. Additionally, the complexity of the supervisor may hinder its application because every candidate controller requires a Kalman filter and a post posterior evaluation ${ }^{[16]}$. Thus, some substitutive methods have been proposed to improve the above-mentioned situation. In [16-19], fuzzy rules based weighting algorithms replaced the probabilistic weighting algorithm. In [1], a new weighting algorithm based on model output error, is proposed. As a result, the long-standing issue, i.e, the closed-loop stability of WMMAC, has been addressed for a class of discrete-time stochastic plant under smooth conditions. The breakthrough result also benefited from the virtual equivalent system (VES) theory ${ }^{[20]}$.

This paper is intended to further improve the convergence rate of weighting algorithm, as well as to adapt the weighting algorithm to time-varying systems such as parameter jump system and slowly time-varying system.

\section{DESCRIPTION OF WMMAC}

Consider the following discrete-time stochastic plant with single input and single output (SISO)

$$
P(k): A\left(k, q^{-1}\right) y(k)=q^{-d} B\left(k, q^{-1}\right) u(k)+\omega(k)
$$

where

$$
\begin{aligned}
A\left(k, q^{-1}\right)= & 1+a_{1}(k) q^{-1}+\cdots+a_{n a}(k) q^{-n a} \\
B\left(k, q^{-1}\right)= & b_{0}(k)+b_{1}(k) q^{-1}+\cdots+b_{n b}(k) q^{-n b} \\
& d \geqslant 1 ; n a \geqslant 1 ; n b \geqslant 1
\end{aligned}
$$

where $y(k), u(k)$ and $\omega(k)$ are the output, input and zero-mean white noise of the system, respectively, $y(k)=0, u(k)=0, \omega(k)=0$ for $k<0$, and that

$$
\lim _{n \rightarrow \infty} \frac{1}{n} \sum_{i=1}^{n}[\omega(i)]^{2}=R<\infty
$$

The plant $P(k)$ can be stable or non-stable, minimum phase or non-minimum phase. Its output $y(k)$ can be rewritten as

$$
y(k)=\phi^{T}(k-d) \theta(k)+\omega(k)
$$

where

$$
\begin{aligned}
\phi^{T}(k-d)= & {[y(k-1), \ldots, y(k-n a), u(k-d), \ldots,} \\
& u(k-d-n b)]
\end{aligned}
$$

$\theta(k)=\left[-a_{1}(k), \ldots,-a_{n a}(k), b_{0}(k), b_{1}(k), \ldots, b_{n b}(k)\right]$

The WMMAC system consists of three components, the model set $\mathbb{M}=\left\{M_{i}, i=1,2, \ldots, N\right\}$, the 'local' controller set $\mathbb{C}=\left\{C_{i}, i=1,2, \ldots, N\right\}$, and the weighting algorithm. The $i^{\text {th }}$ model, controller, and weight are denoted as $M_{i}, C_{i}$, and $p_{i}(k)$, respectively.

The control objective is to track the bounded reference signal $y_{r}(k)<\infty$.

'Local' controllers can be designed according to any existing methods, such as pole assignment, $H^{\infty}$ method, mixed- $\mu$ synthesis tools, PID, etc.

Each 'local' controller $C_{i}$ generates local control $u_{i}(k)$; the 'global' control applied to the plant is obtained by weighting of the local controls, i.e.

$$
u(k)=\sum_{i=1}^{N} p_{i}(k) u_{i}(k)
$$

A simple and direct weighting algorithm is proposed in [1], which depends only on the model output errors. It gets rid of the inconvenience of Kalman filters and off-line membership functions. Details will be given in the next section.

For each model $M_{i} \in \mathbb{M}$, its output is given by

$$
y_{i}(k)=\phi^{T}(k-d) \theta_{i}
$$

where $\theta_{i}$ is the parameter vector of model $M_{i}$. Further, define the output error of each model $M_{i}$, i.e.

$$
e_{i}(k)=y(k)-y_{i}(k)=y(k)-\phi^{T}(k-d) \theta_{i}
$$

As we will see in the next section, $e_{i}(k)$ is used to calculate $p_{i}(k)$. 


\section{WEIGHTING ALGORITHMS WITH CONVERGENCE ANALYSIS}

First, we review a weighting algorithm put forwarded in [1]. Then, a modified weighting algorithm will be proposed in order to improve the convergence rate, as well as to be suitable for time-varying plant.

Weighting algorithm $1^{[1]}$ :

$$
\begin{gathered}
l_{i}(0)=\frac{1}{N} ; p_{i}(0)=l_{i}(0) \\
l_{i}^{\prime}(k)=1+\frac{1}{k} \sum_{p=1}^{k} e_{i}(p)^{2} \\
l_{\text {min }}(k)=\min _{i} l_{i}^{\prime}(k) \\
l_{i}(k)=l_{i}(k-1) \frac{l_{\text {min }}(k)}{l_{i}^{\prime}(k)} \\
p_{i}(k)=\frac{l_{i}(k)}{\sum_{i=1}^{N} l_{i}(k)}
\end{gathered}
$$

Based on Weighting Algorithm 1, a modified version is proposed as follows.

Weighting algorithm 2:

$$
\begin{gathered}
l_{i}(0)=\frac{1}{N} ; p_{i}(0)=l_{i}(0) \\
l_{i}^{\prime}(k)=1+\frac{1}{k} \sum_{p=1}^{k} e_{i}(p)^{2} \\
l_{\text {min }}(k)=\min _{i} l_{i}^{\prime}(k) \\
l_{i}(k)=\frac{l_{\text {min }}(k)}{l_{i}^{\prime}(k)} \\
p_{i}(k)=\frac{l_{i}(k)}{\sum_{i=1}^{N} l_{i}(k)} \\
l_{i}(k-1)\left[\beta_{i}(k)\right]^{c e i l\left(\frac{2}{1-\beta_{i}(k)}\right)}
\end{gathered}
$$

where $\operatorname{ceil}(x)$ is the ceiling function that generates the smallest integer not less than $x$, i.e.,

$$
\operatorname{ceil}(x)=\min \{n \in \mathbb{Z} \mid x \leqslant n\}
$$

To adapt the above mentioned algorithms to slowly time-varying or parameter jump systems, there are two methods to avoid the zero lock-in condition of $p_{i}$ from occurring. One method is to set lower bounds for weights $p_{i}, i=1,2, \ldots, N$ as in [21]; The other is to reset each weight according to (3.6) when the parameters change is detected. In the simulations of this paper, we adopted the latter method. In detail, the numerical value order of $l_{i}(k)$ is used to detect the parameter changes. The related programme is as follows.

$$
\begin{aligned}
& A=\left[l_{1}^{\prime}(k) l_{2}^{\prime}(k) l_{3}^{\prime}(k) l_{4}^{\prime}(k) \ldots\right] \\
& {\left[l_{\min }(k), \operatorname{index}(k)\right]=\min (A) ;}
\end{aligned}
$$

calculates $l_{i}(k)$ according to Weighting Algorithm 2

$$
\begin{gathered}
\text { if } l_{1}(k)=0\left\|l_{2}(k)=0\right\| l_{3}(k)=0 \| l_{4}(k)=0 \cdots \\
\text { if } \quad \operatorname{index}(k)=\operatorname{index}(k-1)
\end{gathered}
$$$$
\text { resets Weighting Algorithm 2; }
$$

end

end

\section{SIMULATION RESULTS}

To verify the effectiveness of the proposed weighting algorithm and the performance of the resulting closed-loop WMMAC system, a lot of simulation results have been conducted with MATLAB ${ }^{\circledR}$ version 6.5. Owing to the space limitation, the corresponding photographes are omitted here. In a word, the weighting algorithm has very good convergence performance under various situations, and the corresponding WMMAC systems have satisfactory stability and tracking performance. 


\section{CONCLUSIONS AND FUTURE WORK}

The stability and convergence of the closed-loop WMMAC system depend only on three conditions, i.e., the stabilizing characteristic of each 'local' controller; the effective model set to cover the uncertainty of plant to be controlled; and the appropriate convergence of the weighting algorithm. Thus, WMMAC provide us with more confidence and flexibility in robust adaptive control practices.

The future research will be focused on two directions. One is to analyze the stability and convergence of WMMAC systems of non-linear timevarying plants. The other is to further relax the convergence conditions of weighting algorithms.

\section{References}

1. W. Zhang, "Stable Weighted Multiple Model Adaptive Control: Discrete-Time Stochastic Plant," Int. J. Adapt. Control and Signal Process, 27, 562-581 (2013).

2. D. T. Magill, "Optimal adaptive estimation of sampled stochastic processes," IEEE Trans. Automat. Contr., 10, 434-439 (1965).

3. D. G. Lainiotis, "Partitioning: aunifying framework for adaptive systems I: Estimation; II: Control," Proc. IEEE, 64, 1126-1143 and 1182-1197 (1976).

4. M. Athans et al., "The stochastic control of the F-8C aircraft using a multiple model adaptive control (MMAC) method-Part I: Equilibrium flight," IEEE Trans. Automat. Contr., 22, 768-780 (1977).

5. D. W. Lane and P. S. Maybeck, "Multiple model adaptive estimation applied to the Lambda URV for failure detection and identification," in Proc. IEEE 33rd Conf. Decision Contr., Lake Buena Vista, FL, 678-683 ( 1994).

6. C. Yu, R. J. Roy, H. Kaufman, and B. W. Bequette, "Multiple-model adaptive predictive control of mean arterial pressure and cardiac output," IEEE Trans. Biomed. Eng., 39, 765-778 (1992).

7. R. L. Moose, H. F. Van Landingham, and D. H. McCabe, "Modeling and estimation for tracking maneuvering targets," IEEE Trans. Aerospace Elec. Syst., 15, 448-456 (1979).

8. X. R. Li and Y. Bar-Shalom, "Design of an interacting multiple model algorithm for air traffic control tracking," IEEE Trans. Contr. Syst. Tech., 1, 186-194 (1993).

9. M. Athans, S. Fekri, and A. Pascoal, "Issues on robust adaptive feedback control," in Preprints 16th IFAC World Congress, Invited Plenary paper, Prague, Czech Republic, 9-39 (2005).

10. Sajjad Fekri, Michael Athans and Antonio Pascoal, "Issues, progress and new results in robust adaptive control," Int. J. Adapt. Control and Signal Process, 20, 519-579 (2006).

11. Sajjad Fekri, Michael Athans and Antonio Pascoal, "Robust multiple model adaptive control (RMMAC): A case study," Int. J. Adapt. Control and Signal Process, 21, 1-30 (2007).

12. Baram Y, "Information, consistent estimation and dynamic system identification," Ph.D. Dissertation, MIT, Cambridge, MA, U.S.A., November (1976).

13. Baram Y, Sandell NR, "An information theoretic approach to dynamical systems modeling and identification," IEEE Transactions on Automatic Control, 23, 61-66 (1978).

14. Baram Y, Sandell NR, "Consistent estimation on finite parameter sets with application to linear systems identification," IEEE Transactions on Automatic Control, 23, 451-454 (1978).

15. Athanasios Kehagias, "Convergence Properties of the Lainiotis Partition Algorithm," Control and Computers, 19, 1-6 (1991).

16. M. Kuipers and P. Ioannou, "Practical Robust Adaptive Control: Benchmark Example," in Proc. American Control Conference, Seattle, Washington, USA, June, (2008).

17. M. Kuipers and P. Ioannou, "Multiple model adaptive control with mixing," IEEE Transactions on Automatic Control, 55, 1822-1836 (2010).

18. Baldi S., Ioannou P. A., and Mosca E., "Multiple Model Adaptive Mixing Control: the Discrete-time case," IEEE Trans. on Automatic Control, 57, 1040-1045 (2012).

19. Nasser Sadati, Guy A. Dumont, and H. R. Feyz Mahdavian, "Robust Multiple Model Adaptive Control Using Fuzzy Fusion," 42nd South Eastern Symposium on System Theory, Tyler, TX, USA, March 7-9 (2010).

20. W. Zhang, "On the Stability and Convergence of Selftuning Control-Virtual Equivalent System Approach," International Journal of Control, 83, 879-896 (2010).

21. J. A. Custafson, P. S. Maybeck, 'Flexible Spacestructure Control Via Moving-Bank Multiple Model Algorithms, "IEEE Transactions on Aerospace and Electronic Systems, 30, 750-757 (1994). 